\title{
ALTERACIONES MUSCULOESQUELÉTICAS Y ADAPTACIONES BIOMECÁNICAS DURANTE LOS TRIMESTRES DE EMBARAZO: UNA REVISIÓN SISTEMÁTICA
}

\author{
MUSCULOSKELETAL CHANGES AND BIOMECHANIC ADAPTATIONS DURING THE \\ THREE TRIMESTERS OF PREGNANCY: A SISTEMATIC REVIEW
}

\author{
Natalia Quesada Salazar ${ }^{1}$ \\ nataqsalazar@gmail.com \\ 1 Universidad de Costa Rica, San José, Costa Rica \\ ${ }^{1}$ Trabajadora independiente, Costa Rica \\ Envío original: 2020-12-01 Reenviado: 2021-01-26, 2021-03-02 Aceptado: 2021-04-19 \\ Publicado: 2021-05-04
}

Doi: https://doi.org/10.15517/pensarmov.v19i1.44961

\begin{abstract}
RESUMEN
El embarazo es un proceso progresivo que involucra cambios de tipo hormonal, mecánico, fisiológico y emocional en la mujer, y que causa que el sistema musculoesquelético se adapte constantemente. Estos cambios, suelen identificarse a través de la postura y la biomecánica de los movimientos en la vida diaria. En esta investigación se realizó una revisión sistemática para encontrar las principales alteraciones y adaptaciones durante el embarazo, incluyendo artículos del 2008 al 2018, con mujeres gestantes sanas, con un solo feto, sin alteraciones musculoesqueléticas previas, de Índice de Masa Corporal (IMC) normal y cualquier metodología de investigación excepto estudios de caso. Se identificaron 13 estudios que refieren evaluar las tres etapas de gestación, edades desde los 20 a los 35 años, así como con diversos objetivos de investigación. La postura de la mujer gestante presenta un aumento de la lordosis lumbar, la curvatura torácica y el ángulo de inclinación anterior de la pelvis. La tarea de sentarse y levantarse de una silla requiere de mayor control del movimiento así como de tiempo de ejecución. La oscilación y las fuerzas de reacción del suelo del Centro de Presión corporal aumentan al estar de pie, lo que resulta en una mayor distancia entre los pies como estrategia de control del equilibrio. Finalmente, la biomecánica de la marcha se adapta a una menor velocidad, longitud de paso y etapa de despegue de los pies del piso, con un aumento del ancho
\end{abstract}


de paso y una mayor base de apoyo. En la gestación, la mujer desarrolla adaptaciones de tipo anatómicas y mecánicas como respuesta a los cambios progresivos experimentados.

Palabras clave: embarazo, revisión sistemática, sistema musculoesquelético, biomecánica.

\begin{abstract}
Pregnancy is a progressive process that involves changes of hormonal, mechanical, physiological, and emotional characteristics in women, and that stimulates the musculoskeletal system to adapt constantly. These changes are usually identified through posture and the biomechanics of day-to-day movements. This study consists of a systematic review of the main alterations and adaptations during pregnancy, including articles from 2008 to 2018 that involved healthy pregnant women, with a single fetus, without previous musculoskeletal affections, with normal BMI, and any methods of study excluding case studies. 13 studies were identified which indicate evaluation of participants with chronological ages from 20 to 35 years, during the three trimesters of pregnancy, as well as different study objectives. Posture in a pregnant woman shows an increase in the lumbar lordosis, the thoracic curvature, and greater anterior tilt of the pelvis. The task of sitting and rising from a chair requires more control to complete the movement as well as increased execution time. Oscillation movement and ground reaction forces of the center of pressure of the body increase during standing posture, which results in a greater distance between the feet as a strategy to control the balance. Finally, gait's biomechanics adapts to a slower speed, smaller step length and less time with feet off the ground, but to a wider step and support base. During pregnancy, women develop anatomic and mechanic adaptations in response to the progressive changes they experience.
\end{abstract}

Key Words: pregnancy, systematic review, musculoskeletal system, biomechanics

\title{
INTRODUCCIÓN
}

El embarazo se compone de una serie de modificaciones progresivas, dentro de las cuales hay una afectación al sistema musculoesquelético que deriva de procesos hormonales, anatómicos y fisiológicos. Estos pueden llegar a influir en distintos aspectos tales como la postura, el patrón de la marcha y el equilibrio, dado a que se dan cambios en la mecánica, derivados del aumento de peso, nueva composición corporal, entre otros (Bey, Arampatzis y Legerlotz, 2018; de Sousa et al., 2018; Forczek, Ivanenko, Bielotowicz y Wacławik, 2018). 
Durante el embarazo estas variaciones pueden dar paso a una cadena de problemas o alteraciones en el cuerpo (Berber y Satılmış, 2020). Entender cómo se dan estos cambios, su frecuencia y el efecto permanente o temporal que se presenta en las gestantes es fundamental para luego entender alteraciones como el dolor durante el embarazo, caídas y otros (Bagwell et al., 2020).

Distintas problemáticas se identifican como comunes durante los procesos de embarazo, como lo son las caídas. Aproximadamente un $25 \%$ de las mujeres experimentan una caída durante la gestación, también el dolor de origen lumbo pélvico y en zonas anatómicas cercanas, donde más del $50 \%$ se queja al menos una vez de dolor de origen musculoesquelético en la zona lumbar. Finalmente, la mujer gestante, experimenta una disminución y falta de promoción de la práctica de actividad física por miedo a riesgos, falta de condiciones adecuadas para la práctica y el desconocimiento acerca de los beneficios del movimiento para el embarazo (Catena, Bailey, Campbell, Stewart y Marion, 2020; Berber y Satılmı, 2020; James et al., 2020).

Adicional a esto, durante ya casi tres décadas los investigadores han tratado de dar explicación a los cambios y adaptaciones generados durante la gestación, como respuesta al constante cambio del centro de gravedad y la ganancia de peso y masa abdominal. Para esto, han concentrado los esfuerzos en la identificación de cambios físicos más comunes entre las mujeres, así como respuestas mecánicas para poder ejecutar movimientos y mantener un balance postural. Esto sin ceder ante la clara ganancia progresiva de tamaño abdominal en un plano frontal hacia anterior.

Durante la última década, posiblemente más, la gestación y particularmente la valoración de las mujeres durante este proceso ha sido de gran interés en la academia. La razón de ser de este interés deriva en la necesidad de comprender como, de manera progresiva y constante el cuerpo es capaz de anticipar o responder a modificaciones corporales derivadas del crecimiento fetal en el útero.

La manera en que el embarazo se ha estudiado en los últimos años ha permitido dar paso a que los investigadores se concentren en el equilibro y la marcha; adicionalmente en cómo en las tres etapas de gestación se ajustan las necesidades del sistema musculoesquelético a los factores internos y externos a los que se expone. Entender cuáles son las alteraciones que se presentan en el embarazo e identificar sus signos en las mujeres gestantes, para los profesionales en la salud, ha sido de gran beneficio ya que permite mejorar la atención dada a esta población y a su vez promueve la investigación continua en este campo.

El propósito de esta revisión es poder extraer la información referente a alteraciones musculoesqueléticas y adaptaciones biomecánicas durante los tres trimestres de embarazo 
dadas en mujeres sanas, así como identificar las características generales de los estudios que documentan estos hallazgos.

\section{METODOLOGÍA}

\section{Diseño}

Este estudio es una revisión sistemática, cualitativa, de carácter descriptivo observacional (Aguilera, 2014). Se da una recopilación de artículos en el periodo de octubre 2008 hasta noviembre de 2018 cuyo espacio corresponde a las bases de datos: Clinical Key, DOYMA, EBSCO Host, EMBASE y ProQuest.

\section{Población}

Se lleva a cabo una revisión y análisis de artículos con restricción de años de publicación, en el idioma español, inglés y portugués. Se agrega a la metodología el uso de los Descriptores en Ciencias de la Salud (DeCS) y Medical Subject Headings (MeSH por sus siglas en inglés) para determinar las palabras clave a utilizar. Se empleó una estrategia de búsqueda utilizando los descriptores en los tres idiomas seleccionados.

Para español: “embarazo", "biomecánica”, "marcha”, "locomoción”, "postura”, "balance postural", "sistema musculoesquelético".

Los mismos, traducidos al idioma inglés: "pregnancy", "biomechanics", "gait", "locomotion", "posture", "postural balance", "musculoskeletal system"

Y finalmente en portugués: "gravidez", "biomecânica”, "marcha”, "locomoção", "postura”, "equilibrio postural", "sistema musculoesquelético"

Se crean dos estrategias de búsqueda utilizando diferentes operadores booleanos. Una inicial que arroja resultados desviados de la línea de investigación y una segunda estrategia que deriva artículos más relevantes y se designa como estrategia final:

[(Embarazo AND Biomecánica AND Marcha OR Locomoción)], [(Embarazo AND Biomecánica AND Balance Postural)] y [(Embarazo AND Sistema Musculoesquelético AND Postura)].

Con esta búsqueda y la adición de bases de datos: PubMed, Scielo, Dialnet, Elsevier y Google Scholar, se establece una muestra final de 13 artículos, con inglés como idioma predominante. Se elimina Clinical Key ya que los resultados no se apegan a las estrategias de búsqueda con DeCS utilizados. Se utiliza la guía PRISMA® (Prefered Reporting Items for 
Systematic Reviews and Meta-Analyses) tomada de Moher, Liberati, Tetzlaff, Altman y The PRISMA Group (2009) para definir la muestra.

\section{Criterios de inclusión y exclusión}

Se incluyeron los estudios que cumplieron con los siguientes criterios: i) participantes mujeres gestantes en los tres trimestres de embarazo, ii) Índice de Masa Corporal (IMC) normal con un embarazo único; de estudios observacionales, ensayos clínicos aleatorios, revisiones bibliográficas, metaanálisis, casos y controles, y estudios de cohortes, con un intervalo de confianza de 95\% y, iii) publicados entre los periodos de octubre 2008 a noviembre 2018

Se excluyeron estudios de caso debido a la baja evidencia científica que estos tienen, cuya población fueran mujeres gestantes con sobrepeso u obesidad, con embarazos gemelares o múltiples y que presentaran alteraciones musculoesqueléticas previas al embarazo. Se definen dos criterios de exclusión redundantes respecto al peso y producto de gestación como parte de los procesos de selección de información.

\section{Riesgo de sesgo}

Procurando mantener la validez de la investigación, se identifican posibles sesgos que pudieran darse en diferentes etapas, principalmente en la etapa de selección de los estudios a revisar; con el fin no de eliminarles sino prevenirles y reducirles (Manterola y Otzen, 2015).

Se seleccionan todos los estudios que cumplieran con los criterios independientemente de sus conclusiones y resultados con el fin de reducir el sesgo de publicación así como se reporta claramente por qué se eliminan y seleccionan los diversos artículos para evitar el sesgo de referencia. Se revisaron cuidadosamente las investigaciones y se hace uso del sistema de referencias bibliográficas Mendeley para evitar sesgo de duplicación. Finalmente, se amplía el lenguaje de búsqueda previniendo el sesgo de idioma y se revisa el nivel de evidencia de las pesquisas para reducir el sesgo de calidad por medio del método Downs y Black adaptado por Forczek et al. (2018); utilizando también los niveles de evidencia y grados de recomendación de acuerdo con la Scottish Intercollegiate Guidelines Network (“SIGN", por sus siglas en inglés). Se obtiene en base con el puntaje final de 17 puntos para Downs y Black un promedio de 11.92 \pm 1.38 para la muestra evaluada.

\section{Proceso de selección y extracción de datos}

La estrategia de búsqueda definida fue utilizada en cada base de datos establecida. Se usó el gestor de referencias Mendeley para filtrar artículos duplicados, así como crear las citas 
bibliográficas de las investigaciones a utilizar. La selección de muestra se llevó a cabo en 4 etapas: duplicados, discriminación por título, por resumen y finalmente por texto completo. A su vez, se hizo una explicación detallada de aquellos artículos que fueron descartados para la revisión.

Las fases de selección se detallan utilizando la guía PRISMA® tanto los estudios incluidos como excluidos (Figura 1). Una vez identificados los artículos para la muestra se procede a extraer la información necesaria para la revisión por medio de un instrumento de recolección de datos específico de los detalles metodológicos de los estudios, aplicando criterios de inclusión y exclusión así como, un instrumento que permitiese identificar y anotar los resultados a extraer respecto a alteraciones musculoesqueléticas y adaptaciones biomecánicas durante los tres trimestres de embarazo (Quesada Salazar, 2019). 


\section{RESULTADOS}

\section{Selección de muestra}

La búsqueda efectuada arrojó un total de 1081 resultados acumulados en todas las bases de datos. De este total se excluyeron estudios por filtros de duplicación, título, resumen y finalmente texto completo. Se realizó un análisis de 13 artículos científicos como muestra final. El control de estudios excluidos se realiza a través de Office Excel para detallar el criterio de exclusión y el dato estadístico para cada etapa de selección. En la Figura 1 se muestran los procesos detallados de selección.
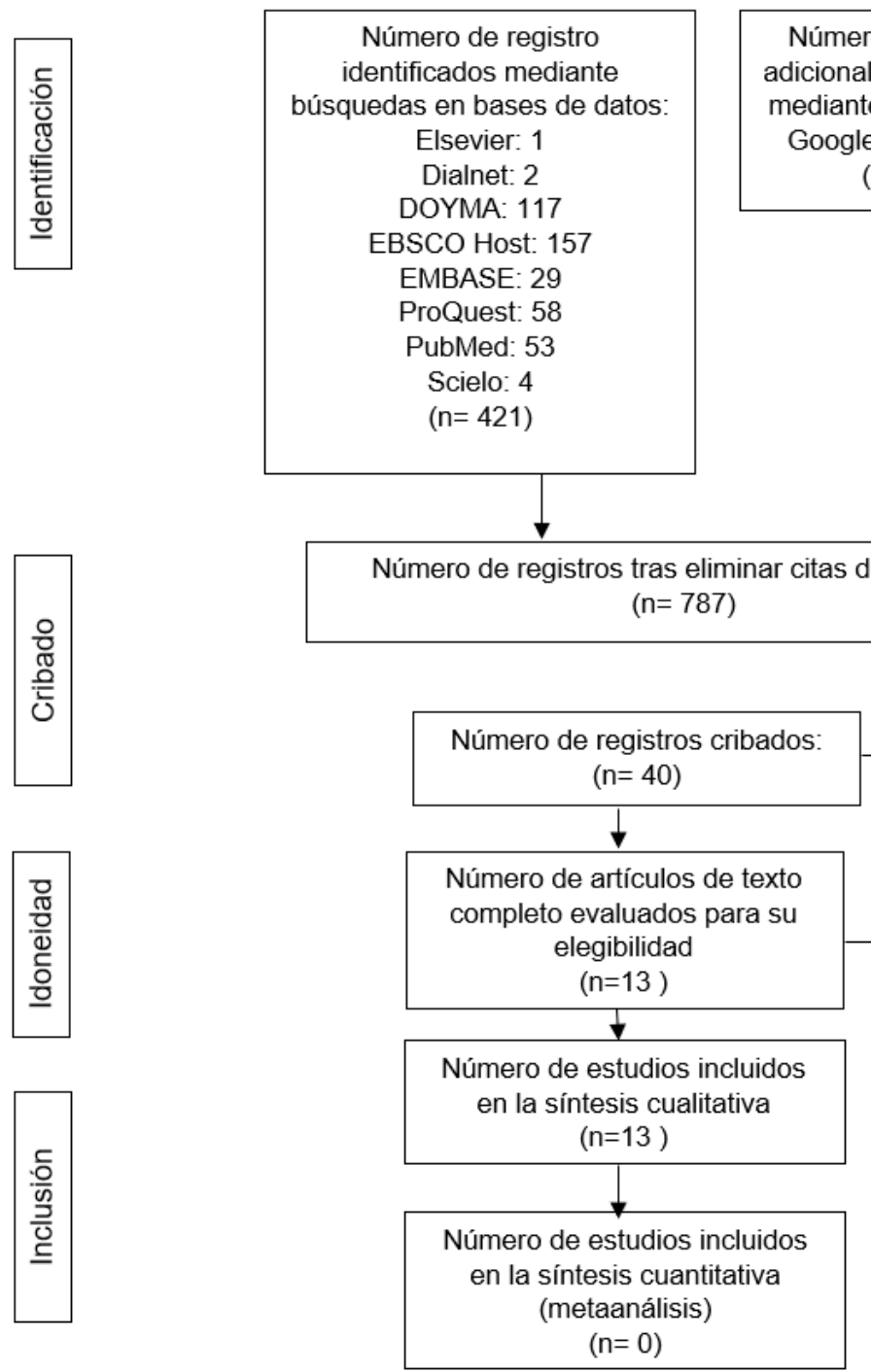

Número de registros adicionales identificados iante otras fuentes: oogle Scholar: 660 $(n=660)$
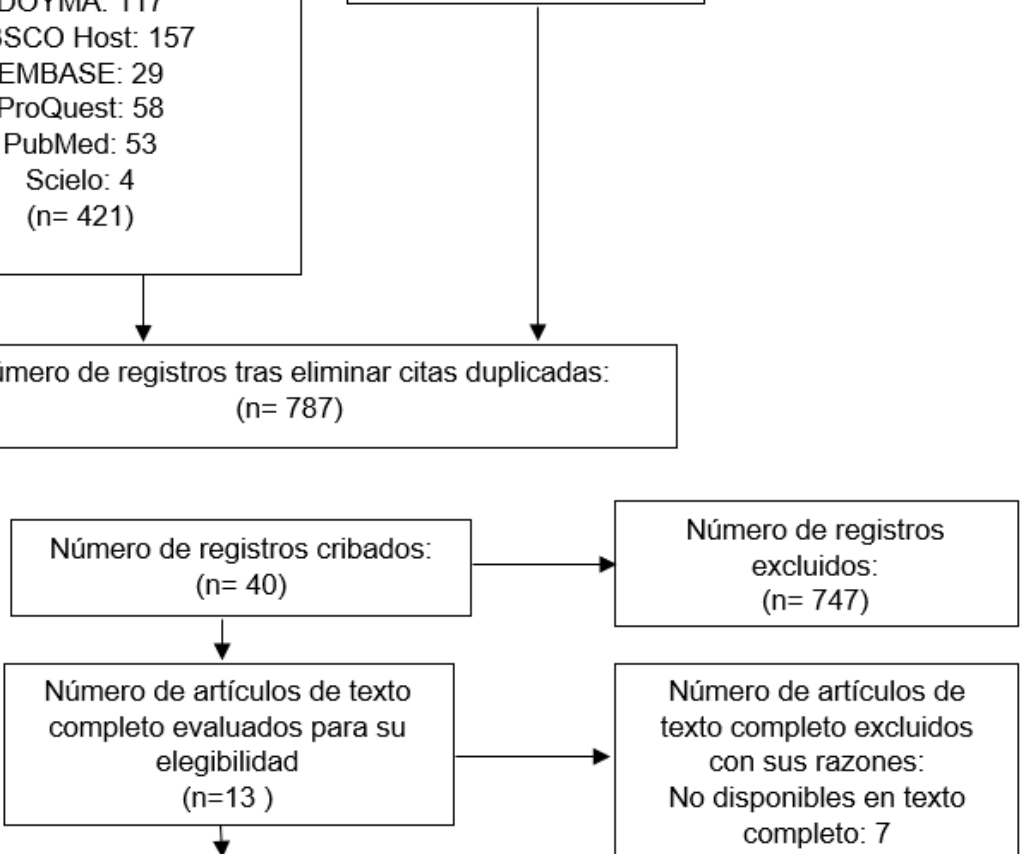
Número de artículos de texto completo excluidos con sus razones: No disponibles en texto completo: 7 Otra población de estudio: 1 Otro objetivo de estudio: 12

Datos irrelevantes a la investigación: 3

Revisiones con duplicados de artículos encontrados en Ios registros: 4 $(n=27)$

Figura 1. Diagrama de flujo de información $P R I S M A ®$ para las fases de selección de la población de estudio de acuerdo con la plantilla de Moher et al. (2009) con traducción tomada de Moher, Liberati, Tetzlaff, Altman y The PRISMA Group (2014, p.177). Elaboración propia de acuerdo con Quesada Salazar (2019, p. 49.), 2020. 


\section{Características de los estudios seleccionados}

La muestra seleccionada se caracterizó por ser predominantemente de idioma inglés, en su mayoría publicados en 2012 y 2013; provenientes de Brasil y Japón principalmente. Todos los estudios muestran un alto grado de recomendación y evidencia científica de acuerdo con las guías utilizadas para evaluar.

La población de estudio se caracteriza por ser mujeres embarazadas y no embarazadas debido a que en la mayoría se trató de casos y controles; con edades entre 20 y 35 años, de promedio entre 26 y 29 años. La edad gestacional reportada corresponde a los tres trimestres de embarazo reportados como trimestres y como semanas de gestación. Se identificaron los grupos de intervención que incluían mujeres gestantes y no gestantes, de 10 a 81 participantes en distintos tamaños dependiendo de los estudios; también describiendo mismas participantes para las distintas mediciones o varios grupos gestacionales simultáneos para las mediciones.

En la Tabla 1 se muestra, en orden cronológico de publicación, un resumen de los estudios seleccionados de acuerdo con: autor (es), participantes de acuerdo con edad en años, edad gestacional y grupos de evaluación, diseño, revista en que fueron publicados e idioma; creada con las características generales de los artículos. 
Tabla 1.

Resumen de características de los artículos seleccionados para la muestra distribuida en orden cronológico de publicación

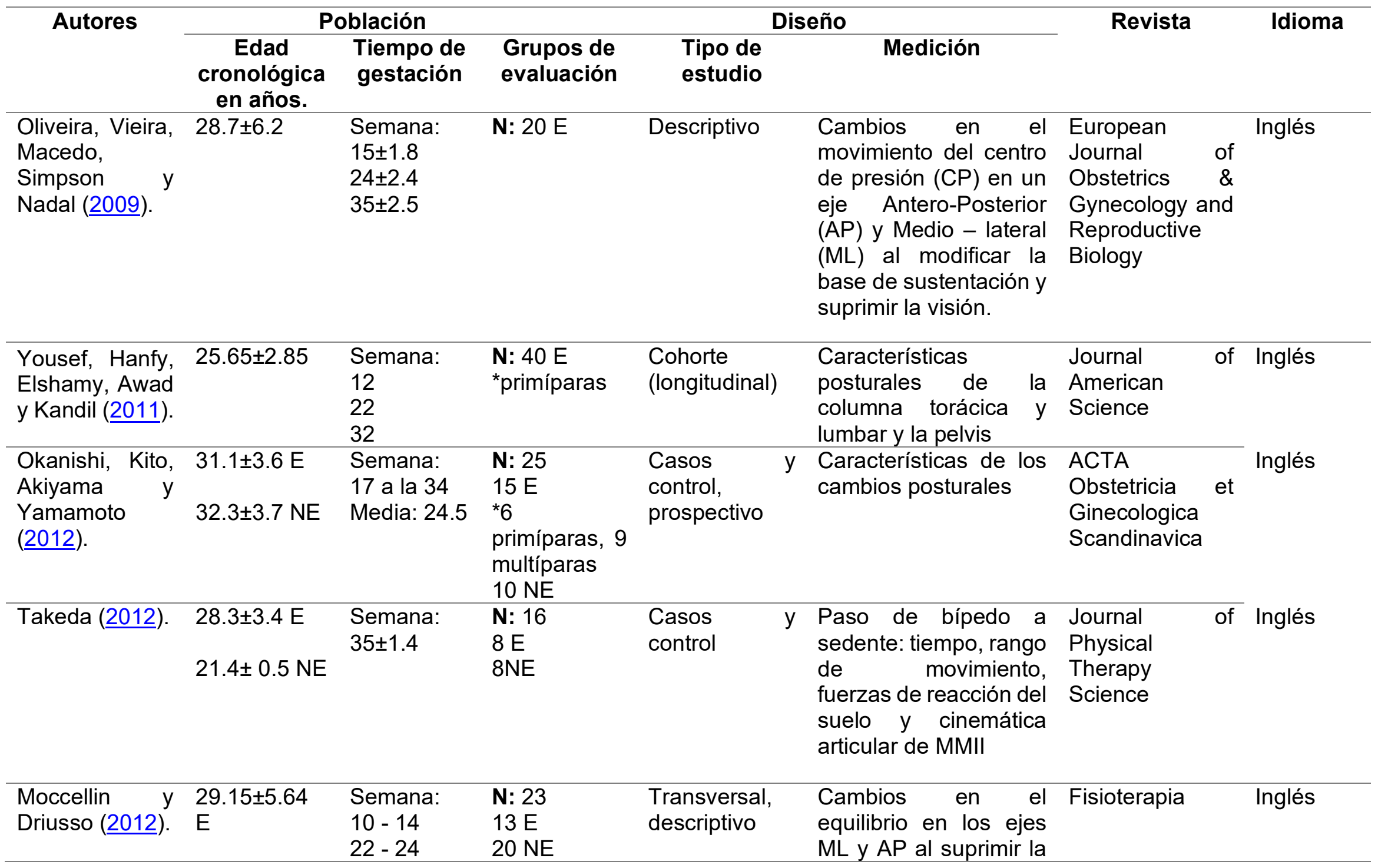




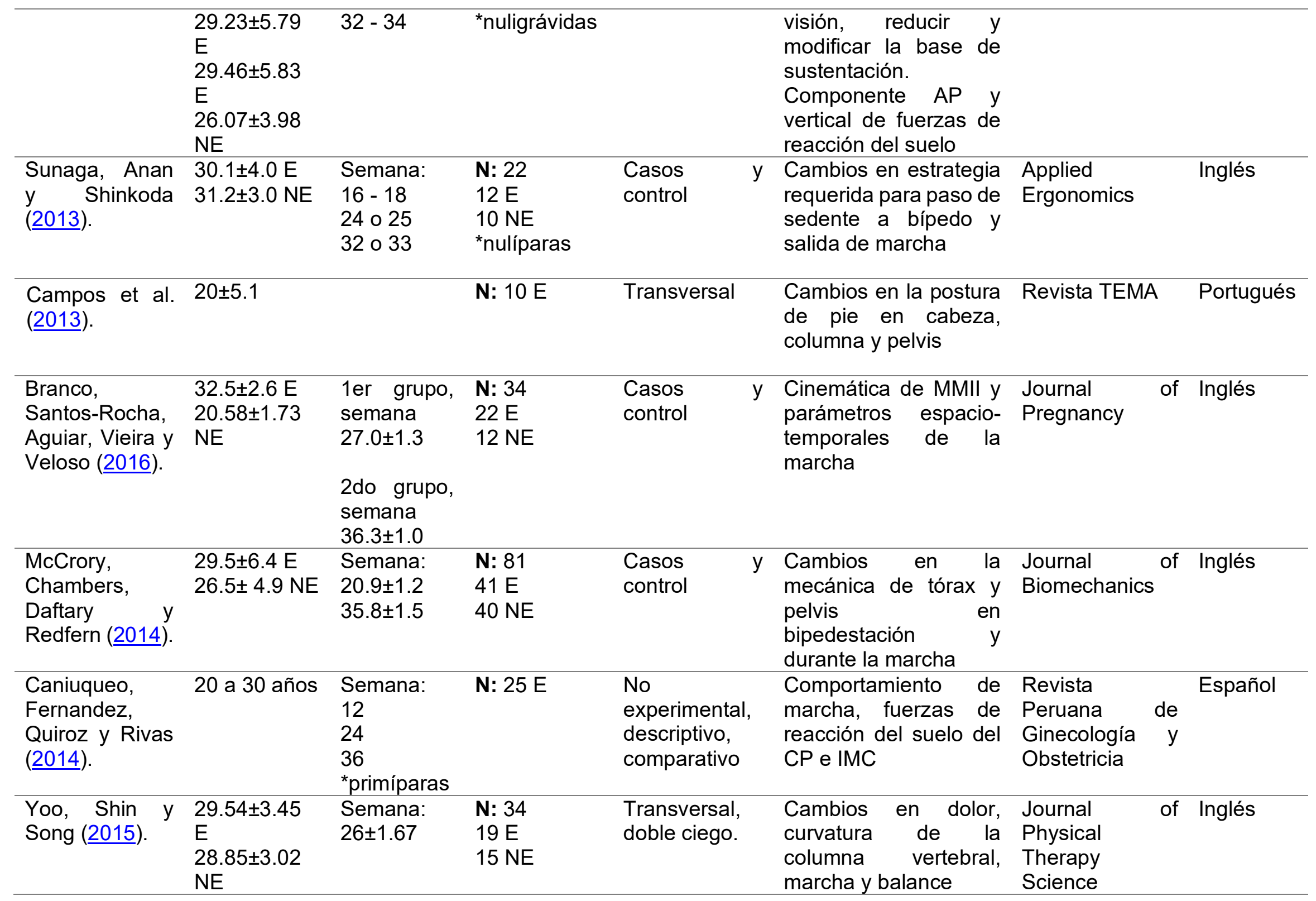




\begin{tabular}{|c|c|c|c|c|c|c|c|c|}
\hline $\begin{array}{l}\text { Sawa et al. } \\
(\underline{2015}) \text {. }\end{array}$ & $\begin{array}{l}30.4 \pm 5.1 \quad E \\
(E T) \\
31.4 \pm 3.2 E \\
(E A)\end{array}$ & $\begin{array}{l}\text { Semana: } \\
17.1 \pm 3.2 \\
(\mathrm{ET}) \\
33.6 \pm 3.1 \\
(\mathrm{EA})\end{array}$ & $\begin{array}{l}\mathbf{N}: 27 \\
16 \mathrm{E} \\
(\mathrm{ET}) \\
11 \mathrm{E} \\
(\mathrm{EA})\end{array}$ & Transversal & & $\begin{array}{l}\text { Características de la } \\
\text { marcha en un eje } \\
\text { vertical, ML y AP }\end{array}$ & Gait \& Posture & Inglés \\
\hline $\begin{array}{l}\text { Danna-Dos- } \\
\text { Santos et al. } \\
(\underline{2018)} .\end{array}$ & $\begin{array}{l}\text { 1. } 28.0 \mathrm{E} \\
\text { 2. } 24.5 \mathrm{E} \\
\text { 3. } 25.0 \mathrm{E} \\
\text { 23.0 NE }\end{array}$ & $\begin{array}{l}\text { Dado por } \\
\text { trimestre: } \\
\text { I trimestre } \\
\text { II trimestre } \\
\text { III trimestre }\end{array}$ & $\begin{array}{l}\text { N: } 40 \\
\text { 1. } 10 \mathrm{E} \\
\text { 2. } 10 \mathrm{E} \\
\text { 3. } 10 \mathrm{E} \\
\text { 10 NE }\end{array}$ & $\begin{array}{l}\text { Casos } \\
\text { control }\end{array}$ & $y$ & $\begin{array}{l}\text { Cambios en el control } \\
\text { postural de acuerdo } \\
\text { con el movimiento del } \\
\text { CP y fuerzas de } \\
\text { reacción del suelo }\end{array}$ & Gait \& Posture & Inglés \\
\hline
\end{tabular}

Nota: Los datos tomados de las poblaciones de cada artículo se anotan de acuerdo con medias de edades en años, medias de semanas gestacionales, excepto para un artículo el cual reporta datos en términos de trimestre; división de grupos de evaluación entre embarazadas y no embarazadas así como, cantidad de participantes por grupo tanto gestantes como no gestantes. Se utilizan las siguientes abreviaturas: N: número de sujetos. E: embarazada, NE: no embarazada, ET: embarazo temprano, EA: embarazo avanzado, ML: medio - lateral, AP: antero - posterior, CP: Centro de presión, MMII para miembros inferiores e IMC para índice de masa corporal. Fuente: elaboración propia con base en los datos recopilados por Quesada Salazar (2019), 2020. 
A continuación se presentan los datos identificados tanto para las alteraciones musculoesqueléticas como adaptaciones biomecánicas y de equilibrio. Se reúnen los estudios de acuerdo con los resultados encontrados y se establecen cuatro grupos.

Grupo A. Estudios con resultados acerca de cambios posturales presentados por: (Yousef et al., 2011; Okanishi et al., 2012; Campos et al., 2013; Yoo et al., 2015; Danna-Dos-Santos et al., 2018).

Grupo B. Estudios con resultados acerca de la ejecución de movimientos: sentarse y levantarse de una silla con pasos reportados por: (Takeda, 2012; Sunaga et al., 2013).

Grupo C. Investigaciones que reportan adaptaciones en el control postural dados por: (Oliveira et al., 2009; Moccellin y Driusso, 2012; Caniuqueo et al., 2014; Yoo et al., 2015; DannaDos-Santos et al., 2018).

Grupo $D$. Artículos de los cuales se extraen datos de adaptaciones biomecánicas en la marcha durante el embarazo: (Branco et al., 2016; McCrory et al., 2014; Caniuqueo et al., 2014; Yoo et al., 2015; Sawa et al., 2015).

Con el fin de examinar adecuadamente todos los resultados encontrados en este estudio, se seleccionaron únicamente los datos que fueran presentados como significativos.

\section{Postura}

El grupo A identifica un aumento de la lordosis lumbar, la curvatura torácica y el ángulo de inclinación pélvica de manera progresiva durante los tres trimestres de embarazo. Okanishi et al. (2012) difieren con datos de una lordosis lumbar y una inclinación posterior de sacro reducida, mientras que para los datos de curvatura torácica no hay alteraciones significativas entre trimestres ni en comparación al grupo no gestante.

Así mismo, este grupo analizado reporta una realineación del tronco superior como efecto del aumento de IMC y tamaño abdominal. Reportan también datos de desviaciones de línea de plomada hacia lado izquierdo tanto de la cabeza como de acromio y, una elevación de escapula hacia la derecha.

\section{Sedente y levantarse}

El grupo B expone que para la ejecución de la sedestación, la mujer gestante requiere de mayor tiempo para llevar a cabo el movimiento, aumenta el tiempo de extensión de rodilla y hay un aumento en las fuerzas de reacción del suelo recibidas para el eje posterior como vertical (Takeda, 2012). Por su parte, llevar a cabo el movimiento de levantarse de la silla evaluado en el I trimestre indica que se requiere de mayor tiempo de levantarse de la silla así como la 
identificación de un menor ángulo de flexión del tronco. Para la cadera, se identifica que durante el I trimestre hay una disminución del ángulo de flexión de esta, mientras que hay un aumento para la extensión de la articulación. El II trimestre, también refleja una disminución de la amplitud del ángulo de la flexión de la cadera.

Finalmente, Sunaga et al. (2013) al comparar las mediciones entre gestantes y no gestantes para el I y II trimestre, determinan que la velocidad de desplazamiento del centro de masa, en un eje horizontal disminuye mientras que a su vez esta velocidad en un eje vertical aumenta.

\section{Control postural}

El grupo C obtiene información acerca de los mecanismos de estabilidad durante el embarazo y adaptaciones que se dan para mantener el control postural ante el paulatino crecimiento abdominal. Anotan que se da un aumento progresivo del balanceo corporal y el área total de oscilaciones, principalmente en el AP. Hay un desplazamiento anterior del centro de gravedad (CG) y un desplazamiento posterior del CM.

La mayoría de las investigaciones estudiadas concuerdan en que tanto la información visual así como la modificación de la base de sustentación, o apoyo, son mecanismos de control de la estabilidad por parte de las gestantes y que definen sus respuestas a fuerzas externas del entorno. Adicional, hacen uso de técnicas de evaluación que permiten encontrar que cerrar los ojos o juntar los pies, van a ser condiciones de mucha demanda de ajustes posturales y energía cada vez más intensa conforme progresa el embarazo. Suprimir la visión o reducir la base de sustento van a generar un aumento de las oscilaciones totales, su fuerza y su área, y a su vez van a aumentar la inestabilidad.

\section{Marcha}

La extracción de información del grupo D permite identificar que las adaptaciones biomecánicas de la marcha en la gestación pueden darse tanto por parámetros espacio temporales como por parámetros de cinética y ajustes articulares.

Para lo espacio - temporal, este grupo de estudios evidencia una disminución de la longitud de paso y zancada, del tiempo de apoyo simple, la cadencia y la velocidad de marcha. A su vez, un aumento del tiempo de doble apoyo y de la base de sustentación que da como resultado un aumento del ancho de paso. Estos se identifican al comparar tanto gestantes con los controles así como entre etapas de gestación. Durante el embarazo estos cambios se hacen más notorios en la última etapa gestacional, y al comparar esta con el I y II trimestre. 
En cuanto a parámetros de cinética y articulaciones. En un eje sagital se reporta una disminución de la magnitud de movimiento de la articulación de cadera al comparar mujeres no gestantes con mujeres en el segundo y tercer trimestre respectivamente. Además, se identifica una clara disminución del pico de movimiento del tobillo para el III trimestre que permite determinar una disminución de la flexión plantar durante esta etapa gestacional.

Para un plano frontal, los autores Branco et al. (2016) anotan un descenso en la magnitud de movimiento para la abducción de la cadera del II al III trimestre de gestación, así como al comparar ambos trimestres con la población de mujeres no gestantes evaluada.

En un eje de valoración AP, hay una disminución en el control del movimiento del tronco al caminar, así como una menor aceleración en la ejecución del movimiento. Por último, se extraen datos de rotaciones de tronco limitadas y menor rotación pélvica en gestantes. Hay una limitación del tórax y una tendencia hacia la extensión de este con menor rango de movimiento en el eje sagital al comparar gestantes avanzadas con los controles.

La muestra evaluada para la presente investigación se caracteriza por la variedad de métodos de medición y metodologías utilizadas en los estudios. A continuación se detalla en la Tabla 2 las técnicas de medición utilizadas por la muestra de este artículo, presentadas de acuerdo con tipo de técnica, variable estudiada y componente evaluado (estático, dinámico o ambos). 
Tabla 2.

Técnicas de medición utilizadas identificadas para la muestra de acuerdo con tipo, variable y componente evaluado

\begin{tabular}{|c|c|c|c|}
\hline Autores & Técnica (s) de medición & Variable dependiente medida & Componente evaluado \\
\hline $\begin{array}{l}\text { Oliveira et al. } \\
(\underline{2009})\end{array}$ & $\begin{array}{ll}\text { - Pruebas } & \text { de } \\
\text { estabilometría } & \text { en } \\
\text { plataforma de fuerza } & \end{array}$ & - Movimiento del Centro de presión (CP) en el eje ML y AP. & $\begin{array}{l}\text { Comportamiento del centro } \\
\text { de presión en la postura de } \\
\text { pie } \\
\text { (E) }\end{array}$ \\
\hline $\begin{array}{l}\text { Yousef et al. } \\
(\underline{2011})\end{array}$. & - Mocap & - Posición anatómica de la columna y pelvis & Postura de pie (E) \\
\hline $\begin{array}{l}\text { Okanishi et al. } \\
(\underline{2012)}\end{array}$ & - Mocap & - Evaluación de la postura y angulación de pelvis & Postura de pie (E) \\
\hline $\begin{array}{l}\text { Moccellin y } \\
\text { Driusso (2012) }\end{array}$ & - Plataforma de fuerza & - Estabilometría en un eje ML y AP & Postura de pie (E) \\
\hline $\begin{array}{l}\text { Sunaga et al. } \\
(\underline{2013})\end{array}$ & - Mocap & $\begin{array}{l}\text { Sedente a bípedo y caminata } \\
\text { - Despegue de la silla } \\
\text { - Primer swing de pierna } \\
\text { - Primer despegue de pierna } \\
\text { - Segundo swing de pierna }\end{array}$ & $\begin{array}{l}\text { Cinemática articular y de } \\
\text { marcha. } \\
\text { Prueba de levantarse de la } \\
\text { silla y caminata (D) }\end{array}$ \\
\hline $\begin{array}{l}\text { Campos et al. } \\
(\underline{2013})\end{array}$ & - Mocap & - Postura de cabeza, columna y caderas & Postura de pie (E) \\
\hline $\begin{array}{l}\text { Branco } \\
(\underline{2016})\end{array}$ et al. & $\begin{array}{l}\text { - Mocap } \\
\text { - Plataforma de fuerza } \\
\text { - Mocap }\end{array}$ & $\begin{array}{l}\text { - Movimiento planar de las articulaciones de MMII } \\
\text { - Patrón de marcha: } \\
\text { - Velocidad, cadencia, tiempo derecho e izquierdo, tiempo } \\
\text { de doble apoyo, tiempo de apoyo y balanceo, ancho de }\end{array}$ & $\begin{array}{l}\text { Postura de pie (E) } \\
\text { Cinemática corporal, } \\
\text { articular y de marcha (D) }\end{array}$ \\
\hline
\end{tabular}




\begin{tabular}{|c|c|c|c|}
\hline & & $\begin{array}{l}\text { paso, ancho de zancada, largo de paso derecho e } \\
\text { izquierdo. } \\
\text { - Ángulos de movimiento para cadera, rodilla y tobillo } \\
\text { bilateral en un plano sagital, frontal y transversal. } \\
\text { - Fuerzas de reacción del suelo en el patrón de marcha }\end{array}$ & \\
\hline \multirow[t]{2}{*}{$\begin{array}{l}\text { McCrory et al. } \\
(\underline{2014})\end{array}$} & - Mocap & $\begin{array}{l}\text { - Posición del tórax y pelvis en plano frontal, sagital y } \\
\text { transversal }\end{array}$ & $\begin{array}{l}\text { Postura y orientación de } \\
\text { tronco y pelvis }(E)\end{array}$ \\
\hline & $\begin{array}{l}\text { - Plataformas de fuerza } \\
\text { - Mocap }\end{array}$ & $\begin{array}{l}\text { - Movilidad del tórax y pelvis en plano frontal, sagital y } \\
\text { transversal } \\
\text { - Ancho y velocidad de paso }\end{array}$ & Cinemática de marcha (D) \\
\hline \multirow[t]{2}{*}{$\begin{array}{l}\text { Caniuqueo et al. } \\
(\underline{2014})\end{array}$} & - Oscilógrafo postural & $\begin{array}{l}\text { - Área, velocidad y energía de desplazamiento del CP en } \\
\text { bípedo }\end{array}$ & Balance postural de pie (E) \\
\hline & - Plataforma de fuerza & $\begin{array}{l}\text { - Fuerzas de reacción del suelo, duración de la pisada } \\
\text { fases y subfases de apoyo y empuje en base a movimiento } \\
\text { del CP }\end{array}$ & $\begin{array}{l}\text { Cinemática corporal y de } \\
\text { marcha (D) }\end{array}$ \\
\hline \multirow[t]{2}{*}{ Yoo et al. (2015) } & $\begin{array}{l}\text { - Plataforma de fuerza } \\
\text { - Mocap }\end{array}$ & $\begin{array}{l}\text { - Curvatura torácica y lumbar } \\
\text { - Movimiento del CP }\end{array}$ & $\begin{array}{l}\text { Postura de pie y balance } \\
\text { corporal }(E)\end{array}$ \\
\hline & $\begin{array}{l}\text { - Mocap } \\
\text { - Plataforma de marcha }\end{array}$ & $\begin{array}{l}\text { - Presión en el paso de la marcha } \\
\text { - Velocidad, cadencia, largo de paso, largo de zancada, } \\
\text { fase de apoyo simple }\end{array}$ & Cinemática de marcha (D) \\
\hline $\begin{array}{l}\text { Sawa et al. } \\
(\underline{2015})\end{array}$ & - Mocap & $\begin{array}{l}\text { - En un eje vertical, ML y AP: } \\
\text { - Movilidad de tronco } \\
\text { - Largo de paso, velocidad de marcha, oscilación corporal } \\
\text { en el paso }\end{array}$ & Cinemática corporal (D) \\
\hline $\begin{array}{l}\text { Danna-Dos- } \\
\text { Santos et al. } \\
(\underline{2018})\end{array}$ & $\begin{array}{l}\text { - Mocap } \\
\text { - Posturografía } \\
\text { plataforma de fuerza }\end{array}$ & $\begin{array}{l}\text { - Fuerzas de reacción del suelo en un plano frontal y sagital } \\
\text { por movimiento de CP } \\
\text { - Área y velocidad de oscilación de CP }\end{array}$ & $\begin{array}{l}\text { Control de la postura de pie } \\
\text { (E) }\end{array}$ \\
\hline
\end{tabular}

Nota. E: estático, D: dinámico, Mocap: Motion Capture o captura de movimiento (Gómez et al., 2018). De acuerdo con Gómez et al. (2018) el término Mocap engloba las técnicas que permiten obtener imágenes de movimiento del cuerpo humano en 2 y 3 D. Esto incluye marcadores en piel, cámaras infrarrojas, cámaras de video, sistemas de análisis de movimiento, entre otros. Cada uno de los artículos de la muestra incluyen variabilidad de instrumentos para cada técnica sin embargo, únicamente se anotan las técnicas de manera general, sin detalle de cómo funcionan o sus características específicas ya que el objetivo es solo ilustrar de qué manera obtuvieron los autores sus resultados. Fuente: elaboración propia con base en los datos recopilados por Quesada Salazar (2019), 2021. 


\section{DISCUSIÓN}

El objetivo de esta investigación era poder revisar la evidencia de alteraciones y adaptaciones que se desarrollan durante el embarazo. Del análisis y extracción de resultados de los artículos seleccionados, se identifica que tanto la marcha como el control postural son los cambios con más incidencia en los resultados; aunque también se estudian movimientos y cambios físicos en la postura.

De acuerdo con los datos extraídos de las características de las pesquisas, los momentos de evaluación durante el embarazo variaron; sin embargo, la mayoría evalúan el III trimestre.

A manera de resumen, las variaciones se dan con predominio en la zona lumbo pélvica mientras que los movimientos específicos de sedestación y bipedestación muestran disminución de velocidad y de amplitud de la tarea. La estabilidad corporal presenta cambios en el control en bípedo, aumento de base de apoyo y adaptaciones más lentas a los factores externos.

Finalmente, la marcha presenta una reducción de la mayoría de los parámetros que involucren aceleraciones tales como cadencia, velocidad y fase de apoyo. Por su parte hay un aumento en la fuerza y demanda muscular necesaria para ejecutar los movimientos tanto de fase de apoyo como de balanceo siempre y cuando no se comprometa el balance. Todos estos son de manera progresiva y en su mayoría se intensifican conforme más semanas de gestación se cumplen.

Los ajustes posturales reportados son congruentes entre los estudios evaluados, así como con Kouhkan, Rahimi, Ghasemi, Naimi y Akbarzadeh Baghban (2015) quiénes identifican valores aumentados tanto para las curvaturas lumbar y torácica como para la inclinación pélvica anterior. Mockridge y Maclennan (2019) mencionan que parte de los cambios posturales en la gestación son derivados de modificaciones fisiológicas hormonales las cuales, por actuar de la relaxina principalmente, favorecen inestabilidades articulares con predominio de la pelvis. Esta hormona, así como los procesos fisiológicos ya mencionados y el crecimiento del útero predisponen a lesiones, limitaciones y alteración del sistema musculoesquelético a medida que se adapta a los cambios (Kesikburun et al., 2018).

Betsch et al. (2015) identificaron cambios torácicos como resultado de su investigación dado por un significativo aumento de esta curvatura, sin embargo no tiene hallazgos al valorar la lordosis lumbar ni la pelvis. A pesar de esto, sí hablan acerca de hallazgos y congruencias en identificar en distintos autores que hay aumento de la lordosis lumbar, la inclinación de la pelvis

y la curvatura o cifosis torácica durante el embarazo, inclusive mantenidos durante el post parto. Este último dato la mayoría de los estudios lo recalcan e inclusive algunos de la muestra de este estudio lo anotan. Es tal el cambio musculoesquelético y anatómico derivado del aumento del 
diámetro abdominal así como el empuje del centro de gravedad hacia anterior por este mismo, que a pesar de que se pierde de manera abrupta, las alteraciones y adaptaciones pueden mantenerse presente semanas, meses y dependiendo de la gravedad o adaptación hasta 1 año post parto.

Si bien esta investigación toma en consideración sólo los cambios durante la gestación, no cabe duda de que hay una diferencia clara entre la ganancia y la pérdida de estas alteraciones. Al comparar la etapa gestacional de 9 meses en la que las modificaciones son progresivas, al parto y post parto donde hay una repentina eliminación de la masa abdominal y el peso cargado, da paso para que el sistema musculoesquelético tarde en volver a adaptarse a las condiciones a las que se le expone.

Betsch et al. (2015) encuentran adicional a estos hallazgos comunes, que, en un plano frontal, la columna presenta una menor desviación hacia lateral con respecto a la línea media espinal; esta última determinada desde la prominencia ósea de la vértebra C7 hasta el punto medio entre los hoyuelos de Venus. De acuerdo con los autores, este dato de desviación lateral de la columna, corresponde a una respuesta corporal de las mujeres gestantes de mantener su cuerpo erguido con el fin de controlar mejor la carga abdomino pélvica.

Biviá-Roig, Lisón y Sánchez-Zuriaga (2018) así como Betsch et al. (2015) se enfrentan a que los resultados obtenidos para comprobar sus hipótesis de investigación no alcanzan a ser significativos para valorar los cambios de la postura durante el embarazo. Sin embargo, BiviáRoig et al. (2018) si concuerdan en dejar claro que no hay homogeneidad en la presentación de hallazgos, dado que algunos autores reportan aumentos en las curvaturas; otros no reportan cambios y otros inclusive reportan una disminución de la lordosis e inclusive una posteriorización del sacro, distinto de lo que se viene anotando. La revisión de resultados del grupo A de la presente investigación, permite identificar la misma limitación en los estudios.

Lo que los investigadores exponen es que esta falta de congruencia entre estudios podría estar derivada no de falta de información a extraer de los grupos poblacionales, sino de la forma en que se extraiga, es decir los métodos y técnicas de evaluación y medición. A mayor detalle de medición y uso de tecnologías de recepción de información, más clara va a ser la alteración identificada en una mujer gestante.

Continuando con el resultado encontrado con más frecuencia entre los estudios, Catena, Bailey, Campbell y Music (2019) reportan, en un análisis de la sedestación en un plano sagital, que hay mayor movilidad espinal derivada de una característica lordosis lumbar propia de la gestación. Los cambios en el plano sagital, identificados durante el movimiento de sedestación, de acuerdo con estos autores mencionados, podrían estar relacionados a los cambios en la 
postura desde la bipedestación particularmente relacionados al aumento de la lordosis así como el aumento de la inclinación anterior de la pelvis por el aumento de la masa en el abdomen conforme hay crecimiento fetal.

Catena et al. (2020) además, identifican que la lordosis lumbar característica durante el embarazo, la cual influencia una inclinación anterior de la pelvis, no sólo se manifiesta en la postura de bipedestación, sino que también, durante la marcha, y por lo tanto resultan en una modificación del patrón mecánico de la locomoción.

Catena, Bailey et al. (2019) encuentran un aumento del trabajo muscular y de la actividad de columna en la ejecución del movimiento de sentarse, predominantemente en la última etapa antes de alcanzar el asiento. Además, encuentran que hay una menor actividad del trabajo muscular de cadera y un aumento del trabajo ejecutado por la articulación de la rodilla.

En cuanto al balance postural, la presente investigación así como la mayoría de las investigaciones identificadas proponen que los cambios en el balance derivan de la concentración de masa abdominal así como la necesidad de compensar los desajustes que esto genera en el cuerpo al estar de pie para las gestantes. Moreira, Elias, Gomide, Vieira y do Amaral (2017) exponen que la oscilación corporal aumenta de manera progresiva conforme avanza la edad gestacional, y que se intensifica al suprimir la visión y reducir la base de sustentación. Moreira et al. (2017) encontraron que hay un aumento del movimiento en un eje AP, en concordancia con los demás autores.

Catena, Campbell, Werner e Iverson (2019) así como los demás autores revisados tratan de justificar las adaptaciones o cambios posturales debido a adaptaciones mecánicas como repuesta a la ganancia de masa abdominal. Sin embargo no tuvieron éxito, más bien identificaron que en un eje AP el CM logró mantenerse consistente sin amplios cambios de movimiento, y que los datos antropométricos no son justamente relacionables a déficits de balance postural.

La marcha durante el embarazo sigue siendo un foco de estudio de amplio interés entre investigadores debido a que llega a parecerse a la marcha de un adulto mayor tal y como lo plantean Forczek et al. (2019). A su vez, se modifica totalmente la mecánica y predispone a caídas o tropiezos en casi un 25\% de las mujeres gestantes (Ersal, McCrory y Sienko, 2014; Jang, Hsiao y Hsiao-Wecksler, 2008). Así como en esta pesquisa se encuentra que hay una adaptación en el trabajo muscular de las articulaciones de MMII, Catena et al. (2020) también identifican un aumento de la flexión de cadera y rodilla al caminar. Forczek et al. (2019) encontraron que los cambios a la distribución plantar en la marcha pueden estar dados como mejoría en la respuesta de la estabilidad lateral durante la marcha; además tiene un origen musculoesquelético de aumento de pronación de pie y ancho de pie por retención de líquidos. 
Además, asocian el aumento en la base de sustentación como método de mantener el balance y prevención de caídas, tal y como lo hacen poblaciones de infantes y adultos mayores. El objetivo final de esta adaptación es poder ajustar el movimiento del centro de gravedad en un espacio mayor y que no permita perder el control de la postura en los movimientos de fase de apoyo simple.

Bagwell et al. (2020) encuentran que los momentos de fuerza de la musculatura de abducción de cadera resultan mayores tanto para mujeres embarazadas como en etapa post parto en comparación a los grupos control. Adicional atribuyen esto a la posibilidad de que el aumento de la movilización del tronco en un plano lateral para la marcha conocida como "waddling" o comúnmente descrita como "marcha de pato" caracterizada por una amplia base, que puede influir en el balance y que podría ser el foco de estudio para entender mejor el comportamiento de la musculatura de cadera en la marcha de la gestante.

Catena, Campbell et al. (2019) encuentran un patrón de disminución de la velocidad así como los demás autores, relacionado inversamente proporcional a la edad gestacional; a mayores semanas de embarazo menor velocidad de marcha. Marshall et al. (2020) también identifica una disminución de valores como cadencia y velocidad principalmente en el III trimestre, como mecanismo de prevención de caídas como de adaptación al desajuste postural por ganancia de peso abdominal.

Wong y McGregor (2018) encuentran que hay un aumento de la base de sustentación nuevamente y de la abducción de cadera, así como mayor tiempo en la fase de doble apoyo todo con el objetivo de favorecer la estabilidad y reducir las fuerzas externas que alteran el equilibrio. Branco, Santos-Rocha, Vieira, Silva, Aguiar y Veloso (2016) encontraron que la ganancia de masa corporal y peso así como la composición de este en cuanto a grasa, permite explicar los cambios en la mecánica y demanda energética muscular durante las tres etapas de gestación.

Las adaptaciones a la biomecánica de los movimientos en el embarazo, en congruencia con los datos extraídos de la muestra, permiten entender el comportamiento muscular en las articulaciones de cadera, rodilla y tobillo de acuerdo con la etapa gestacional. Sin embargo, esta información carece de alta evidencia para entender con mayor detenimiento; brindando un espacio de potencial investigación y campo de estudio para el futuro.

Catena et al. (2020) mencionan que la cinemática corporal en un plano sagital ejecuta dos tareas: tanto mantener el control postural así como desarrollar la mecánica de la propulsión durante la ejecución de la marcha.

Krkeljas (2018) encuentra que las adaptaciones de la marcha tienen una relación directa con el control postural y la estabilidad (p. 318). Las adaptaciones en el ancho y largo de paso 
tienen una estrecha relación con los cambios en los ángulos del CG y el CP. Cuando se da el aumento en el ancho del paso, Krkeljas (2018) identifican un mayor desplazamiento del centro de gravedad en el eje ML. Además encontraron que hay un aumento de casi un $25 \%$ de la inclinación del tronco conforme progresa el embarazo en un eje sagital, y que este tiene una relación directa con el aumento del ancho de paso.

Así como Biviá-Roig et al. (2018), Krkeljas (2018) también concuerda en mencionar que esta disparidad de resultados está fundamentada en la falta de unificación de técnicas de medición y que por lo tanto no permite establecer cambios fijos y congruentes entre sí; a pesar de que en su mayoría los autores concuerdan en sus hallazgos. Wong y McGregor (2018) concuerdan en exponer que la amplitud de definiciones de parámetros tales como ancho de paso o largo de zancada, la multiplicidad de métodos de evaluación y medición, la diferenciación de grupos de valoración, así como de la utilización de controles o mismas etapas gestacionales como referencia pueden influir en la significancia de los datos a obtener, y por lo tanto la congruencia de estos.

Parece ser congruente entre los estudios revisados para esta investigación, así como los que también se han encargado de estudiar a la población de mujeres gestantes que la prioridad es entender cómo se comporta el cuerpo para poder dar respuesta a problemas como dolor lumbopélvico en el embarazo, el equilibrio, la marcha, los ajustes posturales, las caídas, entre otros. Sin embargo, no han podido concretar la metodología ideal que les permita unificar los hallazgos que van obteniendo y sucede, como se pudo identificar en esta investigación que se repiten varias veces los mismos artículos y resultados entre autores dando explicación a diversas adaptaciones y no así nuevas investigaciones con parámetros y mediciones distintas que permitan dar paso a nuevos datos y clarificar las dudas aún presentes al evaluar a esta población particular.

Es posible identificar, por medio de 13 artículos de esta muestra, que hay una gran variedad de métodos e instrumentos de evaluación al plantear las metodologías de los estudios. Si bien, se identificaron como más recurrentes en la presente investigación las plataformas de fuerza y equipos Mocap, la realidad es que a nivel de metodologías de investigación y técnicas de medición hay una gran variedad de métodos utilizados. Esta falta de homogeneidad metodológica para una sola hipótesis a comprobar va a seguir influyendo en la calidad de evidencia científica que se obtenga para diversos factores evaluables en la población de mujeres gestantes. 


\section{LIMITACIONES}

Se identifica en el presente estudio la limitación de falta de congruencia en las técnicas de medición revisadas por lo que no todos los resultados obtenidos para una misma variable $u$ objetivo de medición son congruentes u homogéneos, puesto que no se está trabajando con los mismos componentes ni características.

\section{CONCLUSIONES}

Las mujeres gestantes durante el embarazo, definido por tres trimestres, experimentan cambios en su anatomía que dan paso a alteraciones musculoesqueléticas de predominio de tronco y columna para responder al aumento de peso corporal y masa abdominal por el crecimiento fetal y movimiento uterino. Adicional, esto predispone a establecer adaptaciones en la biomecánica de los movimientos esenciales como tener control postural y habilidad de marcha siempre con la prioridad de mantener el control del peso y movimiento del centro de gravedad y de masa evitando caídas y movilizándose de manera controlada.

En general, la mayoría de las investigaciones presentan y detallan hallazgos y resultados claros, congruentes y significativos al hacer estudios entre gestantes y al comparar con controles. Sin embargo, hay algunos autores que difieren de los resultados que se identifican y en su mayoría lo justifican a la falta de unificación de medidas de investigación, de modalidad de evaluación y de establecer claras definiciones de los valores a medir.

Se identifica la importancia de ahondar aún más en el campo de las adaptaciones y alteraciones en el embarazo, para terminar de entender movimientos esenciales como la marcha y poder dar paso a nuevas investigaciones de tareas de la vida diaria que sean acorde a poblaciones gestantes de diversas sociedades y poder definir cómo afecta el embarazo actividades de mayor complejidad. Adicional, se vuelve fundamental empezar a diferenciar las investigaciones de acuerdo con poblaciones de mujeres a nivel mundial en cuanto a etnia.

\section{REFERENCIAS}

Aguilera, R. (2014). ¿Revisión sistemática, revisión narrativa o metaanálisis?. Revista de la Sociedad Española del Dolor, 21(6), 359-360. doi: https://doi.org/10.4321/S113480462014000600010

Bagwell, J. J., Reynolds, N., Walaszek, M., Runez, H., Lam, K., Smith, J. A., y Katsavelis, D. (2020). Lower extremity kinetics and muscle activation during gait are significantly different 
during and after pregnancy compared to nulliparous females. Gait \& Posture, 81, 33-40. doi: https://doi.org/10.1016/j.gaitpost.2020.07.002

Berber, M. A., y Satılmıs, İ. G. (2020). Characteristics of Low Back Pain in Pregnancy, Risk Factors, and Its Effects on Quality of Life. Pain Management Nursing, 21(6), 579-586. doi: https://doi.org/10.1016/j.pmn.2020.05.001

Betsch, M., Wehrle, R., Dor, L., Rapp, W., Jungbluth, P., Hakimi, M., y Wild, M. (2015). Spinal posture and pelvic position during pregnancy: a prospective rasterstereographic pilot study. European Spine Journal, 24, 1282-1288. doi: https://doi.org/10.1007/s00586-014-3521-6

Bey, M. E., Arampatzis, A., y Legerlotz, K. (2018). The effect of a maternity support belt on static stability and posture in pregnant and non-pregnant women. Journal of Biomechanics, 75(25), 123-128. doi: https://doi.org/10.1016/j.jbiomech.2018.05.005

Biviá-Roig, G., Lisón, J. F., y Sánchez-Zuriaga, D. (2018). Changes in trunk posture and muscle responses in standing during pregnancy and postpartum. PLOS One, 13(3), e0194853. doi: https://doi.org/10.1371/journal.pone.0194853

Branco, M., Santos-Rocha, R., Aguiar, L., Vieira, F., y Veloso, A. (2016). Kinematic Analysis of Gait in the Second and Third Trimesters of Pregnancy. Journal of Mechanics in Medicine and Biology, 16(4), 1-9. doi: https://doi.org/10.1142/s021951941650055x

Branco, M., Santos-Rocha, R., Vieira, F., Silva, M. R., Aguiar, L., y Veloso, A. P. (2016). Influence of body composition on gait kinetics throughout pregnancy and postpartum period. Scientifica, 2016. doi: https://doi.org/10.1155/2016/3921536

Campos, G. B., Carvalho, M., de Sousa, T., Neves, L. M. B., Dantas, I., Pequeno, T., y Menezes, D. R. (2013). Alterações posturais em gestantes: uma análise através da biofotogrametria computadorizada. Revista Tem@, 14(20-21), 102-116. Recuperado de http://revistatema.facisa.edu.br/index.php/revistatema/article/view/165

Caniuqueo, A., Fernandes, J., Quiroz, G., y Rivas, R. (2014). Cinética de marcha, balance postural e índice de masa corporal durante el primer, segundo y tercer trimestre de embarazo. Revista Peruana de Ginecología y Obstetricia, 60(2), 109-115. Recuperado de http://www.redalyc.org/html/3234/323431582003/

Catena, R. D., Bailey, J. P., Campbell, N., y Music, H. E. (2019). Stand-to-sit kinematic changes during pregnancy corrrespond with reduced sagittal plane hip motion. Clinical Biomechanics, 67, 107-114. doi: https://doi.org/10.1016/j.clinbiomech.2019.05.014

Catena, R. D., Campbell, N., Werner, A. L., e Iverson, K. M. (2019). Anthropometric changes during pregnancy provide little explanation of dynamic balance changes. Journal of Applied Biomechanics, 35(3), 232-239. doi: https://doi.org/10.1123/jab.2018-0345 
Catena, R. D., Bailey, J. P., Campbell, N., Stewart, B. C., y Marion, S. J. (2020). Correlations between joint kinematics and dynamic balance control during gait in pregnancy. Gait \& Posture, 80, 106-112. doi: https://doi.org/10.1016/j.gaitpost.2020.05.025

Danna-Dos-Santos, A., Magalhães, A. T., Silva, B. A., Duarte, B. S., Barros, G. L., Silva, M. D. F. C., ... Cardoso, V. S. (2018). Upright balance control strategies during pregnancy. Gait \& Posture, 66, 7-12. doi: https://doi.org/10.1016/j.gaitpost.2018.08.004

Ersal, T., McCrory, J. L. y Sienko, K. H. (2014). Theoretical and experimental indicators of falls during pregnancy as assessed by postural perturbations. Gait \& Posture, 39(1), 218-223. doi: https://doi.org/10.1016/i.gaitpost.2013.07.011

Forczek, W., Ivanenko, Y. P., Bielotwicz, J., y Wacławik, K. (2018). Gait assessment of the expectant mothers - Systematic Review. Gait \& Posture, 62, 7-19. doi: https://doi.org/10.1016/j.gaitpost.2018.02.024

Forczek, W., Ivanenko, Y., Curyło, M., Frązcek, B., Masłon, A., Salamaga, M., y Suder, A. (2019). Progressive changes in walking kinematics throughout pregnancy - a follow up study. Gait \& Posture, 68, 518-524. doi: https://doi.org/10.1016/j.gaitpost.2019.01.004

Gómez, L. L., Jaramillo, A. M., Ruiz, M. A., Velásquez, S. M., Páramo, C. A., y Silva, G. J. (2018). Sistemas de captura y análisis de movimiento cinemático humano: una revisión sistemática. Prospectiva, 16(2), 24-34. doi: https://doi.org/10.15665/rp.v16i2.1587

James, P., Morgant, R., Merviel, P., Saraux, A., Giroux-Metges, M. A., Guillodo, Y., Dupré, P.F., y Muller, M. (2020). How to promote physical activity during pregnancy: a systematic review. Journal of Gynecology Obstetrics and Human Reproduction, 49(9), 101864. doi: https://doi.org/10.1016/j.jogoh.2020.101864

Jang, J., Hsiao, K. T. y Hsiao-Wecksler, E. T. (2008). Balance (perceived and actual) and preferred stance width during pregnancy. Clinical Biomechanics, 23(4), 468-476. doi: https://doi.org/10.1016/j.clinbiomech.2007.11.011

Kesikburun, S., Güzelküçük, Ü., Fidan, U., Demir, Y., Ergün, A., y Tan, A. K. (2018). Musculoskeletal pain and symptoms in pregnancy: a descriptive study. Therapeutic advances in musculoskeletal disease, 10(12), 229-234. doi: https://doi.org/10.1177/1759720X18812449

Kouhkan, S., Rahimi, A., Ghasemi, M., Naimi, S. S., y Akbarzadeh Baghban, A. (2015). Postural changes during first pregnancy. Birtish Journal of Medicine \& Medical Research, 7(9), 744753. doi: https://doi.org/10.9734/BJMMR/2015/16730 
Krkeljas, Z. (2018). Changes in gait and posture as factors of dynamic stability during walking in pregnancy. Human Movement Science, 58, 315-320. doi: https://doi.org/10.1016/j.humov.2017.12.011

Manterola, C., y Otzen, T. (2015) Los sesgos en investigación clínica. International Journal of Morphology, 33(3), 1156-1164. Recuperado de https://scielo.conicyt.cl/pdf/ijmorphol/v33n3/art56.pdf

Marshall, M. R., Montonye, A. H. K., Conway, M. R., Schlaff, R. A., Pfeiffer, K. A., y Pivarnik, J. M. (2020). Longitudinal changes in walking cadence across pregnancy and postpartum. Gait \& Posture, 79, 234-238. doi: https://doi.org/10.1016/i.gaitpost.2020.05.012

McCrory, J. L., Chambers, A. J., Daftary, A., y Redfern, M. S. (2014). The pregnant "waddle": An evaluation of torso kinematics in pregnancy. Journal of Biomechanics, 47(12), 2964-2968. doi: https://doi.org/10.1016/j.jbiomech.2014.07.009

Moccellin, A. S., y Driusso, P. (2012). Adjustments in static and dynamic postural control during pregnancy and their relationship with quality of life: A descriptive study. Fisioterapia, 34(5), 196-202. doi: https://doi.org/10.1016/j.ft.2012.03.004

Mockridge, A., y Maclennan, K. (2019). Physiology of pregnancy. Anaesthesia \& Intensive Care Medicine, 20(7), 397-201. doi: https://doi.org/10.1016/..mpaic.2019.05.001

Moher, D., Liberati, A., Tetzlaff, J., Altman, D. G., y The PRISMA Group. (2009). Preferred reporting items for systematic reviews and meta-analyses: The PRISMA statement. PloS Medicine, 6(7), e1000097. doi: https://doi.org/10.1371/journal.pmed.1000097

Moher, D., Liberati, A., Tetzlaff, J., Altman, D. G., y The PRISMA Group. (2014). Ítems de referencia para publicar revisiones sistemáticas y meta-análisis: la declaración PRISMA. Revista Española de Nutrición Humana y Dietética, 18(3)172-181. doi: https://doi.org/10.14306/renhyd.18.3.114

Moreira, L. S., Elias, L. A., Gomide, A. B., Vieira, M. F., y do Amaral, W. N (2017). A longitudinal assessment of myoelectric activity, postural sway, and low-back pain during pregnancy. Acta of Bioengineering and Biomechanics, 19(3), 77-83. doi: https://doi.org/10.5277/ABB00753-2016-02

Okanishi, N., Kito, N., Akiyama, M., y Yamamoto, M. (2012). Spinal curvature and characteristics of postural change in pregnant women. Acta Obstetricia et Gynecologica Scandinavica, 91(7), 856-861. doi: https//doi.org/10.1111/j.1600-0412.2012.01400.x

Oliveira, L. F., Vieira, T. M. M., Macedo, A. R., Simpson, D. M., y Nadal, J. (2009). Postural sway changes during pregnancy: A descriptive study using stabilometry. European Journal of 
Obstetrics Gynecology \& Reproductive Biology, 147(1), 25-28. doi: https://doi.org/10.1016/j.ejogrb.2009.06.027

Quesada Salazar, N. (2019). Alteraciones musculoesqueléticas, adaptaciones biomecánicas y métodos utilizados para medición durante los tres trimestres de embarazo. Revisión bibliográfica. 2008-2018 (Tesis de Licenciatura). San José, Costa Rica. Recuperado de http://repositorio.sibdi.ucr.ac.cr:8080/jspui/bitstream/123456789/9008/1/44327.pdf

Sawa, R., Doi, T., Asai, T., Watanabe, K., Taniguchi, T., y Ono, R. (2015). Differences in trunk control between early and late pregnancy during gait. Gait \& Posture, 42(4), 455-459. doi: https://doi.org/10.1016/j.gaitpost.2015.07.058

de Sousa, V. P., Dantas, V.J., Lira, S. O., Cury, A. Mediros, C. N., y Viana, E. S. (2018). Gait variables comparison between pregnant women with and without lumbopelvic pain. Brazilian Journal of Pain, 1(3), 212-216. doi: https://doi.org/10.5935/2595-0118.20180042

Sunaga, Y., Anan, M., y Shinkoda, K. (2013). Biomechanics of rising from a chair and walking in pregnant women. Applied Ergonomics, 44(5), 792-798. doi: https://doi.org/10.1016/j.apergo.2013.01.010

Takeda, K. (2012). A Kinesiological Analysis of the Stand-to-Sit during the Third Trimester. Journal of Physical Therapy Science, 24(7), 621-624. doi: https://doi.org/10.1589/jpts.24.621

Wong, J. K. L., y McGregor, A. H. (2018). Spatiotemporal gait changes in healthy pregnant women and women with pelvic girdle pain: A systematic review. Journal of Back and Musculoskeletal Rehabilitation, 31(5), 821-838. doi: https://doi.org/10.3233/BMR-170828

Yoo, H., Shin, D., y Song, C. (2015). Changes in the spinal curvature, degree of pain, balance ability, and gait ability according to pregnancy period in pregnant and nonpregnant women. Journal of Physical Therapy Science, 27(1), 279-284. doi: https://doi.org/10.1589/jpts.27.279

Yousef, A. M., Hanfy, H. M., Elshamy, F. F., Awad, M. A., y Kandil, I. M. (2011). Postural Changes during Normal Pregnancy. Journal of American Science, 7(6), 1013-1018. doi: https://doi.org/10.7537/marsjas070611.157 